\title{
L'Environnement Numérique d'Apprentissage inscrit dans le paradigme de la modélisation systémique de la complexité
}

\author{
Modeling of Next Generation Digital Learning Environments: Complex
}

Systems Theory

\author{
Marc Trestini ${ }^{1}$ \\ ${ }^{1}$ Laboratoire Lisec, Université de Strasbourg, France, marc.trestini@unistra.fr
}

RÉSUMÉ. Les outils technologiques de l'information et de communication permettent d'accéder facilement à de vastes quantités de connaissances humaines. La connaissance est utilisée pour autonomiser les personnes et les enrichir culturellement et matériellement et pour construire une société durable. Les réseaux sociaux, des OCW, les MOOC et les approches connectivistes de l'apprentissage contribuent largement à l'appropriation de nouvelles connaissances par un large public. Mais l'analyse du fonctionnement de ces Environnements numériques d'apprentissage (ENA) et des processus en jeu dans l'acquisition et le partage des connaissances devient de plus en plus complexe. Le recours à la modélisation des systèmes complexes permet néanmoins de transcrire les phénomènes observés et d'en faciliter l'analyse à l'aide d'outils spécifiques. Cet article présente et met en lumière l'apport de la théorie des systèmes complexes à l'étude du comportement d'ENA de nouvelle génération. Il décrit en détail une démarche réaliste et propose des outils de pilotage efficaces.

ABSTRACT. Communication information technology tools provide easy access to vast amounts of human knowledge. Knowledge is used to empower and enrich people culturally and materially and to build a sustainable society. Social networks, OCWs, MOOCs and connectivist approaches to learning contribute significantly to the construction of this knowledge. But the study of these new digital learning environments (ENA) is also becoming more complicated. The use of complex system modelling makes it possible to transcribe the observed phenomena and facilitate their analysis using specific tools. This paper presents and highlights the value of the complex systems theory for the study of the newgeneration ENA behaviour. It describes in depth a realistic approach and proposes effective management tools.

MOTS-CLÉS. Environnement numérique d'apprentissage, MOOC, activité instrumentée, modélisation des systèmes complexes, paradigme systémique, connectivisme, cognition distribuée, phénomènes émergents.

KEYWORDS. Digital learning environment, MOOC, instrumented activity, complex systems modeling, system paradigm, connectivism, distributed cognition, emerging phenomena.

\section{Introduction}

Avec l'émergence des réseaux sociaux, des MOOC, de l'apprentissage informel via les réseaux et des approches connectivistes de l'apprentissage, l'analyse des environnements numériques d'apprentissage (ENA) est devenue de plus en plus complexe. Le recours à la modélisation devient alors utile car elle permet précisément de transcrire des phénomènes complexes et de faciliter l'analyse des processus en jeu à l'aide d'outils spécifiques. Une fois cette transcription construite, le phénomène perçu complexe peut être étudié plus facilement et ramené à son contexte d'origine. Comme le disait Paul Valéry " nous n'accédons à nos raisonnements que par les modèles que nous nous sommes construits » et «nous ne raisonnons que sur des modèles » [VAL 75, p. 835]

Les premières recherches en technologies éducatives s'appuyaient déjà sur des modèles. Celui qui représente la «structure de l'activité médiatisée » de Vygotski [VYG 85] ou celui qui illustre la «relation individuelle médiatisée » de Kuutti [KUU 96] se sont avérés utiles pour représenter des situations «simples » d'apprentissage médiatisées [PER 05] par les TIC $^{1}$ (Fig. 1). Il se focalise sur la

${ }^{1}$ Technologie de l'Information et de la Communication 
médiation individuelle de l'activité par des artefacts (outils) entre le sujet et son objet. Cette représentation symbolise parfaitement les actions d'un individu poursuivant un projet (finalité de l'activité) et dont l'action est médiatisée par des instruments [LEW 98, p.17].
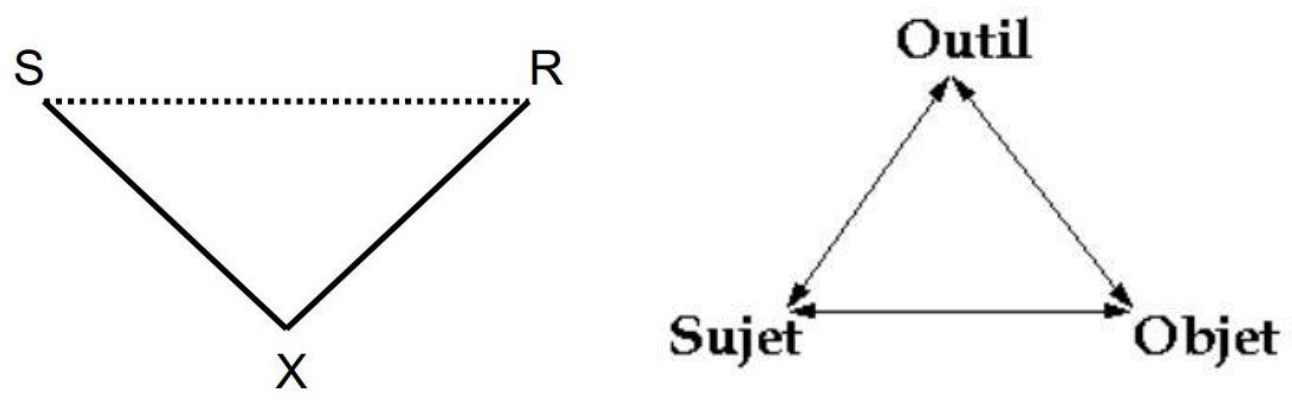

Figure 1. Relation individuelle médiatisée, [VYG 85] à gauche, [KUU 96] à droite

Le modèle d'Engeström [ENG 87] a ensuite permis d'étudier d'autres relations dans des dispositifs qui mettent en scène plusieurs apprenants, en prise avec des artefacts, et placés dans des situations d'apprentissage collaboratif. Il s'est avéré utile pour étudier les tensions ou contradiction à l'intérieur des différents pôles ${ }^{2}$ du système d'activité ou entre ces pôles (Fig. 2).

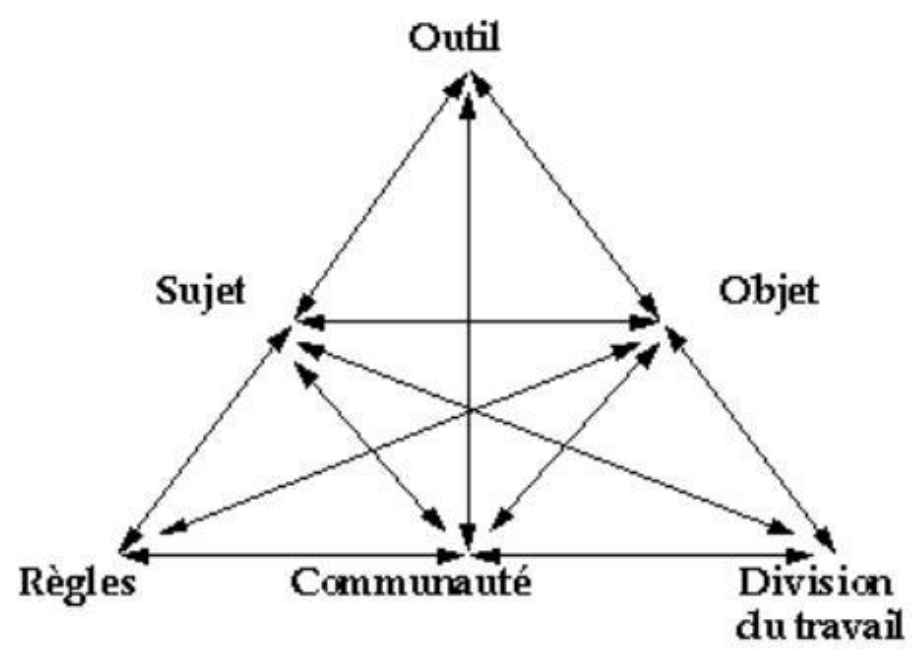

Figure 2. Le modèle d'Engeström [ENG 87]

Ces environnements d'apprentissage se caractérisent en effet par leur «nature composite, articulant des éléments numériques et non numériques » [PER 14, p. 5]. Perçus en outre comme des systèmes d'activités instrumentées, ils sont généralement étudiés le cadre de la de la Théorie de l'activité ou TA [VYG 85 ; LEO 81 ; ENG 87 ; KUU 96 ; RAB 95 ;...]. « La TA est une théorie qui étudie l'homme en tant qu'acteur situé dans un contexte socioculturel et historique » [NAR 96]. Elle a donné lieu à trois principales écoles de pensée : l'école russe, l'école scandinave et l'école française [DAN 05]. Selon cette théorie, «les actions sont toujours insérées dans une matrice sociale composée d'individus et d'artefacts $[\ldots]$ ces derniers lient les individus et les actions d'une manière perméable, changeante et évènementielle » [DUC 05, p. 3].

${ }^{2}$ Sujet, Objet, Outil, Communauté, Règles, Division du travail, 


\section{Limites des modèles utilisés et changement de paradigme}

Si les versions actuelles de cette théorie prennent des formes variées [DAN 05], elles partagent toutes la tentative explicite d'appréhender l'activité dans sa globalité, et leur préoccupation principale est de repenser les distinctions supposées entre les événements et leurs contextes, le discours et l'action, l'aspect social et l'aspect technique [BLA 95]. Nous allons voir en quoi cette approche conjonctive est importante (ne pas séparer ces éléments mais plutôt chercher à les réunir, les assembler). Dans la TA, les artefacts lient les individus et les actions d'une manière perméable, changeante et évènementielle. Ainsi, la prise en compte du contexte et de l'artefact pour la compréhension de l'activité et de l'interaction collective est le caractère fondamental de la théorie de l'activité. En cela, elle nous a permis d'analyser de nombreux dispositifs socio-techniques en action.

Mais dès qu'il s'agissait d'entrer dans des considérations plus fines comme celles qui consistent par exemple à étudier les interactions entre les artefacts didactiques, pédagogiques et techniques [RAB 95 ; MAR 11], que nous supposions constitutifs du pôle «Outil », ou entre différentes « Communautés » d'un même $\mathrm{ENA}^{3}$ (communauté des parents, des enfants, des enseignants, etc.), nous avions le sentiment d'être confronté aux limites des modèles utilisés jusqu'ici et avons été conduit à les transformer pour les adapter à nos besoins [TRE 10a]. Nous avons alors développé en «grappes » chacun des pôles ou constituants du modèle d'Engeström (Fig. 3), dupliqué et étiré dans les trois dimensions de l'espace sa structure interne et ajouté de nouvelles relations entre ses constituants (Fig. 4). Ces différentes « expansions » du modèle de base nous ont alors permis de mettre en évidence d'autres relations entre de nouveaux constituants, intégrés aux pôles existants, et suseptibles d'être, à leur tour, à l'origine de tensions ou contradiction à l'intérieur même des pôles du modèle.

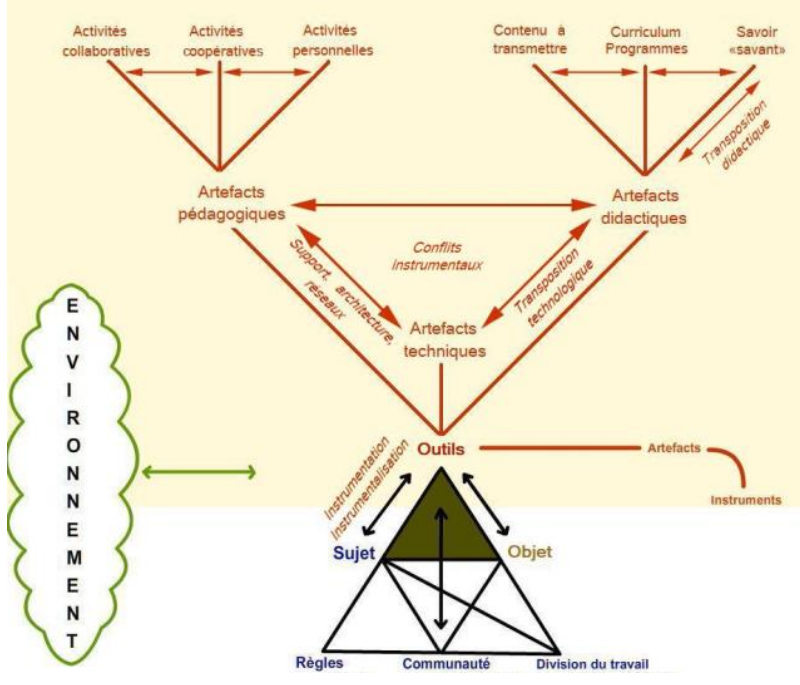

Figure 3. Expansion du pôle "Outil" ( [TRE 10a]

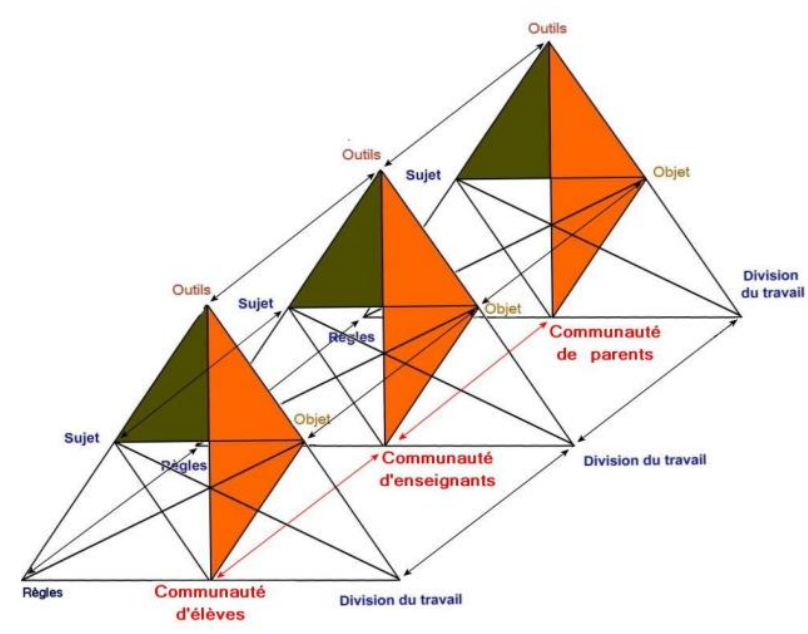

Figure 4. Développement dans l'espace [TRE 10a]

Puis s'est ajouté à ce premier sentiment d'incomplètude du modèle triadique, celui d'avancer à «contre-courant » de ce que la recherche sur la Modélisation Systémique nous enseigne aujourd'hui dans son effort contemporain d'explicitation : raisonner en conjoignant plutôt qu'en disjoignant [LEM 99].

La structure même du modèle d'Engeström, constituée de ses différents pôles, quoique pratique dans de nombreux cas d'analyse, implique aussi, dans l'art de raisonner, l'adoption d'une logique disjonctive propre à la modélisation analytique (MA), laquelle s'oppose avec la logique conjonctive que prône la modélisation systémique (MS) et que la TA revendique. En justifiant une découpe

\footnotetext{
${ }^{3}$ Nous avons à plusieurs reprises étudié les interactions entre ces communautés au cours d'études portant sur le cahier de texte numérique intégré dans des ENT scolaires.
} 
potentiellement infinie des constituants-objets du système, la logique disjonctive conduit progressivement le modélisateur à s'éloigner de la forme canonique du « Système Général » ${ }^{4}$. Adopter une logique conjonctive, c'est précisément éviter la simplification abusive et parfois risquée de la modélisation analytique. Pour Le Moigne, «la simplification du compliqué appliquée au complexe a pour conséquence une aggravation de la complexité » [LEM 99]. Reconnaissons par exemple que la variété socio-culturelle des inscrits à un MOOC (liée à son ouverture) est difficilement «représentable » dans un modèle triadique comme celui d'Engeström. Pour mettre en évidence cette caractéristique particulière, nous pourrions par exemple poursuivre notre décomposition du pôle «sujets» en y ajoutant des «sujets apprenants», des «sujets curieux»,.., des « sujets francophones », etc. Aux constituants du pôle «Outil», déjà composés d'artefacts didactique, pédagogique et tehnique, pourrait aussi s'ajouter un artefact social en sus, comme le suggère d'ailleurs FEL [17]. Mais l'apparition dans le modèle d'Engeström de ces différentes composantes socioculturelles permettrait-elle de mieux représenter un MOOC et son ouverture à un large plublic ?

On observe par ailleurs, dans cette rapide évolution des dispositifs socio-techniques, que les ENA de dernière génération ont un comportement pour le moins chaotique, empruntant en cela les caractéristiques des systèmes complexes. Dès lors, leurs effets apparaissent imprévisibles. Concrètement, cela signifie qu'il n'est pas possible de prédire, par le calcul (un algorithme par exemple), l'issue des processus en jeu. Les résulats d'un apprentissage semblent par exemple bien plus difficile à estimer dans un MOOC que dans un dispositif traditionnel d'EAD, ne serait-ce que par la difficulté que nous aurions à estimer les prérequis des apprenants dans le premier dispositif. Mais chaotique ne signifie pas hasardeux. Les trajectoires des systèmes qui relèvent de cette théorie convergent généralement vers des zones appelées attracteurs. Les attracteurs sont importants parce qu'ils représentent l'ensemble des possibilités d'évolution d'un système. L'apprentissage pourrait représenter un de ces attracteurs ou encore un phénomène émergent, autre caractéristique d'un système complexe, comme la formation de l'eau et de la glace, l'apparition de la vie, l'irruption de la pensée et la genèse d'institutions (langage, monnaie). À partir d'une modélisation systémique dite «de la complexité », il devient alors possible d'élaborer des scénarios plausibles sur la base de l'analyse des données récoltées (trace de l'activité, phénomènes d'émergences, tendances lourdes, etc.). Pour cette raison et toutes celles évoquées précédemment, nous posons l'hypothèse que le paradigme de la modélisation systémique de la complexité peut être avantageusement utilisé pour représenter et analyser un ENA de dernière génération.

Une fois que nous aurons précisé ce que l'on entend précisément par Théorie des systèmes complexes, nous reviendrons sur les fondements des sciences des systèmes et de la complexité avant d'aborder plus spécifiquement le paradigme de la Modélisation systémique de la complexité. Nous choisirons alors de l'adopter pour modéliser les environnements numériques d'apprentissage de dernière génération.

\section{Théorie des systèmes complexes et cadres théoriques de référence}

Après la théorie de la relativité et de la physique quantique, la théorie des systèmes complexes (et du Chaos) est probablement la troisième révolution scientifique majeure du xxe siècle. Elle bouleverse notre façon de raisonner, de percevoir le monde et de prédire les effets de tel ou tel événement sur l'évolution d'un système, autrement dit la manière dont on fait des sciences. On qualifie d'ailleurs ces dernières de sciences des systèmes ou sciences de la complexité. Ces nouvelles sciences «prennent leur statut scientifique au sein du paradigme des épistémologies constructivistes ; alors que les sciences normales se réfèrent habituellement plus volontiers au paradigme des épistémologies positivistes » [LEM 99, p. 22]. Les perspectives constructivistes « prennent le contre-pied du positivisme en insistant

\footnotetext{
${ }^{4}$ « Système Général » ici considéré comme un concept.
} 
sur le rôle du sujet dans l'élaboration de la connaissance et sur l'impossibilité de dissocier les faits des modèles d'interprétation » [LEG 04, p. 70].

Le point commun entre ces différentes «visions constructivistes» repose sur l'idée que la connaissance des phénomènes résulte d'une construction effectuée par le sujet. L'image qu'a ce dernier de la réalité ou les notions qui structurent cette image sont « le produit de l'esprit humain en interaction avec cette réalité, et non le reflet exact de la réalité elle-même » ${ }^{5}$.

\subsection{Définition d'un système complexe}

Un système complexe est avant tout un système. De fait, il est constitué d'un ensemble d'éléments en interaction entre eux et avec l'environnement dans le but de produire les services correspondants à sa finalité. Ils possèdent naturellement les propriétés formelles qui caractérisent un système en général (totalité, rétroaction, équifinalité, etc.). Ce propriétés ont été pour la plupart définies dans la « Théorie générale des systèmes » formalisée après la seconde guerre mondiale par Von Bertalanffy [VON 56]. Le mot complexe vient quant à lui du latin complexus qui signifie «ce qui est tissé ensemble » et de complecti «ce qui contient des éléments différents» [FOR 05, p. 16]. On reconnait à ces systèmes complexes d'autres propriétés que celles des systèmes en général : " ce sont des systèmes ouverts et hétérogènes dans lesquels les interactions sont non linéaires et dont le comportement global ne peut être obtenu par simple composition des comportements individuels [ANI 06, p. 3]. Conscient qu'une définition unanimement acceptée de la nature complexe d'un système reste encore à formaliser, revenons néanmoins sur quatre caractéristiques des systèmes complexes qui font généralement consensus ${ }^{6}$ lors qu'il s'agit de les qualifier ; nous déférencierons ensuite la complexité algorithmique de la complexité naturelle :

- la nature de ses constituants (leur type, leur structure interne);

- son aspect ouvert et chaotique (influences extérieures à différentes échelles) ;

- ses caractéristiques auto-organisatrices et homéostatiques (résilience) dominées par les couplages et les rétroactions (interactions non linéaires, souvent de différents types);

- sa capacité d'évolution vers de nouveaux systèmes à propriétés émergentes et imprévues » [MIT 09].

\subsubsection{Variété des composants et des liaisons}

Les constituants d'un système complexe sont de taille variée, de nature hétérogène (individus, artefacts, symboles...) et incertaine (évolution possible dans le temps) et possèdent une structure interne. "Un système complexe ne se comporte pas comme un simple agrégat d'éléments indépendants, il constitue un tout cohérent et indivisible » [TAR 86, p. 33]. Cette propriété formelle renvoie au concept de totalité. "Les deux notions qui caractérisent la complexité "sont la variété des éléments et l'interaction entre ces éléments". Un système complexe est constitué par une grande variété de composants organisés en niveaux arborescents. Les différents niveaux et composants sont reliés entre eux par une grande variété de liaisons » [Ibid., p. 33]. Et c'est bien la variété des interactions entre ses constituants et la variété des constituants eux-mêmes qui sont à l'origine de l'évolution globale des systèmes complexes.

\footnotetext{
5. « Constructivisme (épistémologie) », Wikipédia (2016, mise à jour 20 mars), disponible à l'adresse : https://fr.wikipedia.org/wiki/Constructivisme (\%C3\%A9pist\%C3\%A9mologie).

${ }^{6}$. Discours d'ouverture du Congrès international sur les systèmes complexes (International Conference on Complex Systems ICCS'12), organisé en hommage au défunt professeur Lorenzo Ferrer Figueras de l’Université de Valence, Espagne, disponible à l'adresse : http://aes.ues-eus.eu/aes2012/allocution ICCS12.pdf.
} 


\subsubsection{Un système complexe n'est pas un système compliqué}

Un système complexe est par ailleurs de nature imprévisible. Il ne permet donc pas de prédire, par le calcul, aussi poussé soit-il, l'issue des processus ou phénomènes mis en jeu ${ }^{7}$. Mais complexe ne signifie pas compliqué. « Nous sommes par exemple capables de comprendre et de prévoir quand et pourquoi un moteur de voiture tombe en panne alors que nous sommes incapables de comprendre et de prévoir exactement l'ensemble des facteurs qui influencent un changement ou un mouvement sociétal (exemple pour une révolution)»[BEG 13]. Lorsque le système est compliqué, nous sommes en capacité de trouver une ou plusieurs solutions, car un système dit "compliqué » est constitué d'un ensemble de choses simples. Il suffit d'un peu de rigueur, de connaissance et de temps pour comprendre son fonctionnement. La méthode générale consiste d'abord à décomposer et isoler chacun des éléments (en composants simples ou en notions simples) pour être ensuite capable de les rassembler. «Le fonctionnement mécanique d'une horloge ou d'un moteur de voiture, par exemple, peut nous sembler compliqué mais nous sommes capables d'expliquer clairement comment ça marche car nous savons quelle incidence a un composant mécanique ou électronique sur l'autre » [Ibid.]. Pour illustrer la différence entre un système complexe et un système compliqué, Snowden [SNO 02, p. 13] fait pertinemment remarquer que «lorsque des rumeurs de réorganisation apparaissent dans une organisation, le système complexe humain commence à muter de façon imprévisible et des tendances se dessinent en prévision du changement. À l'opposé, lorsque l'on s'approche d'un avion, une boîte à outils à la main, rien ne change $! »^{8}$.

\subsubsection{Système ouvert : entre complexité et chaos}

Le système complexe est aussi un système « ouvert », c'est-à-dire en relation permanente avec son environnement. De fait, «il subit des perturbations externes qui sont a priori imprévisibles et inanalysables. Ces perturbations, qui se produisent dans l'environnement, provoquent des adaptations du système qui le ramènent à un état stationnaire » [SNO 02, p. 32]. Pour celui qui 1'étudie, un système fermé est plus prévisible qu'un système ouvert dans le sens où étant totalement isolé des influences externes, il ne sera soumis qu'à des modifications internes et se retrouvera dans un certain nombre d'états définis par ses conditions initiales. Et si ces dernières sont invariables quel que soit le nombre d'expériences, autrement dit le nombre de fois que l'on observe le comportement du système, de sa phase initiale à sa phase finale (ce qui est très difficilement réalisable), il se comportera comme un automate qui déroule un programme connu. En ce sens, il ne peut être considéré comme complexe, aussi compliqué que ce programme puisse d'ailleurs être ! La connaissance des différents états intermédiaires étant suffisante pour définir le système, son évolution et sa finalité en deviennent prévisibles et prédictibles. Mais c'est bien la difficulté à garantir l'invariance des conditions initiales appliquées à un système fermé qui en fait un système ouvert et donc un système potentiellement complexe (car le caractère ouvert est une condition nécessaire mais non suffisante pour qu'un système soit complexe).

\subsubsection{Sensibilités aux conditions initiales}

En son temps, Edward Lorenz soulevait la difficulté de telles prévisions lorsqu'il cherchait à prédire des événements météorologiques à long terme. Grâce à des systèmes informatiques, et fort de sa connaissance des lois déterministes de Galilée et de Newton (également dites prévisionnistes) selon lesquelles les conditions initiales permettraient de déterminer l'état futur d'un système, il s'était donné pour objectif de prédire la météo à partir de simulations informatiques fondées sur un modèle mathématique. En 1963, ses travaux ont révélé le fait que des variations infimes entre deux situations initiales pouvaient conduire à des situations finales sans rapport entre elles. Il affirma ainsi qu'il n'était

\footnotetext{
${ }^{7}$. Si cette prédiction est impossible par le calcul, elle le devient de manière probabiliste avec d'autres outils tels que ceux de la modélisation systémique de la complexité. Nous le verrons dans le paragraphe qui suit.

8. Traduction de l'auteur.
} 
pas envisageable de prévoir correctement les conditions météorologiques à très long terme (par exemple un an) parce qu'une incertitude d'un millionième lors de la saisie des données de la situation initiale pouvait conduire à une prévision totalement erronée. L'« effet papillon » est une métaphore célèbre qui illustre bien ce constat. S'inscrivant dans la «théorie du chaos », elle vise à décrire le phénomène fondamental de sensibilité aux conditions initiales. Cette métaphore est parfois simplement exprimée à l'aide d'une question : «Le battement d'ailes d'un papillon au Brésil peut-il provoquer une tornade au Texas ? » Cette sensibilité pourrait se traduire par le constat suivant : quel que soit le niveau de précision sur les conditions initiales, le système évoluera quand même de façon totalement imprévisible.

\subsubsection{Système complexe ou chaotique?}

La sensibilité aux conditions initiales n'est pas la seule cause responsable de la difficulté à prévoir le comportement d'un système complexe. Dans un système ouvert, les influences réciproques entre l'activité du système, son environnement et ses finalités modifient continuellement sa structure. Les «bruits aléatoires », issus de l'environnement ou des constituants du système, sont actifs sur les relations qui lient ces derniers. Ces événements difficilement contrôlables (caractère aléatoire du bruit) sont autant de perturbations qui contribuent à modifier le système. Qu'ils soient internes ou externes, les bruits modifient ce dernier en le faisant évoluer vers des formes nouvelles et le plus souvent inconnues. Que ces systèmes soient sociaux ou matériels, naturels ou artificiels, il est très difficile de prédire leur comportement et leur évolution car le monde dans lequel nous vivons est par nature principalement chaotique et il ne nous permet que très rarement d'établir les équations des systèmes que nous voulons étudier.

Une autre raison pour laquelle il est difficile de prévoir le comportement d'un système complexe est donc la méconnaissance des règles qui nous disent comment le système va changer, autrement dit l'impossibilité de modéliser ce système par des équations prédictives solvables.

Mais alors, à quoi bon étudier un système complexe si l'on ne peut en aucune manière prédire son comportement futur? La réponse se trouve dans le titre de l'ouvrage d'Henri Atlan [ATL 79] : Entre le cristal et la fumée. L'auteur tente par cette métaphore de situer la complexité entre ces deux extrêmes : le modèle parfaitement connu du cristal et celui imprévisible de la fumée [Ibid., p. 5].

\subsubsection{Complexité alogoritmique et complexité naturelle}

On peut distinguer la complexité algorithmique de la complexité naturelle. La première est celle que l'on peut traiter avec un ordinateur, un programme ou un algorithme. Le but à atteindre est connu, aussi complexe soit-il. La finalité est précisée et détermine la valeur de la procédure. La complexité naturelle est inhérente aux «systèmes non totalement maîtrisés par l'homme parce que non construits par lui » [ATL 79, p. 5] : systèmes biologiques comme la mémoire, les systèmes sociaux, etc. Cette distinction est fondamentale dans la mesure où la théorie de l'auto-organisation que nous allons évoquer ne trouve sa justification que dans des systèmes à complexité naturelle. Ces derniers sont un mélange de savoir et d'ignorance. Ils constituent une sorte de «compromis entre deux extrêmes: un ordre répétitif parfaitement symétrique dont les cristaux sont les modèles physiques les plus classiques et une variété infiniment complexe et imprévisible dans les détails comme celle des formes évanescentes de la fumée » [Ibid.]. Simon (1974) montrait déjà en 1969 la différence d'intelligibilité entre les objets naturels et les objets artificiels. Les objets naturels sont, selon lui, analysés avec l'hypothèse que «la complexité est tissée de simplicité ; la complexité correctement analysée est seulement un masque dissimulant la simplicité »; il conviendrait donc, toujours selon lui, de trouver la forme ordonnée qui se cache dans l'apparent désordre. La théorie des fractales, introduite en 1975 par Benoît Mandelbrot, en serait une illustration récente. «L'Institut de Santa $\mathrm{Fe}^{9}$ sera créé en 1984 pour tenter d'étudier les

\footnotetext{
${ }^{9}$. http://www.santafe.edu/.
} 
conditions par lesquelles c'est parfois l'ordre qui émerge du chaos, ce qui constitue très exactement le contraire d'un effet papillon. ${ }^{10}$

\section{Argument en faveur de l'application de cette théorie à la modélisation d'ENA}

Avoir pour projet de modéliser un monde de connaissance, tel un ENA de dernière génération, en se référant au paradigme constructiviste de la modélisation systémique de la complexité, peut paraitre un exercice audacieux. Et postuler de surcroît que toute acquisition de connaissance peut s'accommoder d'un monde non nécessairement construit dans le seul but d'apprendre l'est davantage encore. Nous voulions pourtant relever ce défi en appréhendant l'organisation cognitive et le système cognitif humain comme des systèmes complexes auto-organisateurs capables d'apprendre au sein d'un monde organisé dans ce but (plutôt que construit au sens formel du terme), en conjuguant deux courants de pensée dont l'union semblait, il y a encore quelques années, utopique. Argumentons ici ce choix dont le développement principal se trouve (à la demande de Jean-Louis Le Moigne) dans le «Cahier des Lectures MCX (Modélisation de la Complexité)»du «Réseau Intelligence de la Complexité » [TRE 09b].

Le premier de ces courants est fondé sur la théorie des systèmes complexes qui postule que toute occasion d'apprentissage résulte de la désorganisation d'un système sujet à des perturbations extérieures. Que ce système soit social ou cognitif, il se réorganise ensuite en augmentant son degré de variété ou de complexité.

«En tant que système, il s'enrichit de cette désorganisation grâce à un processus d'apprentissage non dirigé, au sens où cet apprentissage ne résulte nullement d'un programme préétabli dans la mémoire humaine ou dans l'environnement naturel ou social de cette mémoire. [...] La cause efficiente de cet apprentissage réside dans la rencontre aléatoire du système de la mémoire et de certains facteurs de bruit en provenance de son environnement. [...] Le produit de cet apprentissage consiste en une succession de catégories psychologiques toujours plus fines ou différenciées, dont la liste et le mode de construction sont susceptibles d'être remis en cause à n'importe quelle étape du processus. » [ANC 92].

On peut comprendre intuitivement ce phénomène lorsque l'on pense à la théorie classique de l'évolution: «Les mutations, qui sont précisément des erreurs de réplication des ADN, sont considérées comme la source de l'augmentation progressive de la diversité et de la complexité des êtres vivants » [ATL 98]. L'augmentation de la complexité d'un système peut donc produire un effet positif sur eux ; elle devient source d'apprentissage. Mais cette théorie tient également pour acquis que ce processus auto-organisateur ne peut opérer que dans des systèmes à complexité naturelle, c'est-àdire une complexité inhérente aux «systèmes non totalement maîtrisés par l'homme parce que non construits par lui » [ATL 79, p. 5], tels les systèmes biologiques comme la mémoire, les systèmes sociaux, etc.

Le second courant s'intéresse au contraire à la manière de «construire » ces mondes, d'où l'apparent paradoxe. Par leur nature prescriptive, les modèles de design d'enseignement (ou Instructional Design) s'appuient en effet essentiellement sur des approches épistémologiques de type béhavioriste dans le but d' 'instruire» le sujet. À l'inverse, les environnements constructivistes d'apprentissage mettent l'emphase sur des environnements interactifs qui sollicitent l'expérience personnelle du sujet, d'abord au travers d'activités collectives, soutenues par le formateur et le groupe social, et ensuite lors d'activités individuelles. L'approche connectiviste va encore au-delà en se référant principalement aux processus métacognitifs, voire méta-métacognitifs auxquels les apprenants doivent accéder pour apprendre en réseau [SIE 05 ; BAT 77]. Dans ces derniers cas, l'apprentissage

\footnotetext{
10. « Effet papillon », Wikipédia (2016, mise à jour 31 août), disponible à l'adresse : https://fr.wikipedia.org/wiki/Effet_papillon\#cite note-6.
} 
n'est plus tributaire d'un «enseignement» stricto sensu. Formellement, dans les modèles constructivistes, voire connectivistes, le processus d'apprentissage opère indépendamment des situations cognitives construites par l'enseignant ou par le concepteur. Au plus, ces environnements requièrent de la part de ce dernier la «création » d'environnements « réels » utilisant le contexte dans lequel l'étude est appropriée [JON 91]. Puisqu'il s'agit là de concevoir «artificiellement» des situations «réelles" d'apprentissage ou de simuler des situations liées à un contexte naturel, les modèles de design d'enseignement se sont progressivement accommodés des croyances et pratiques constructivistes. Aux paradigmes fondateurs des modèles de design d'enseignement, d'abord béhavioriste, puis béhavioriste et cognitiviste, vient maintenant s'ajouter le paradigme constructiviste [TRE 08].

De notre point de vue, une des clés du succès de l'«application de la théorie des systèmes complexes à la modélisation d'ENA de nouvelle génération » réside dans l'articulation réussie entre ces deux courants. Pour Lemire [LEM 08, p. 38], construire un monde de connaissance, c'est d'abord «tisser des liens entre des démarches de modélisation de la réalité et le système général ». C'est une manière d'expliquer le monde perçu comme un système qui évolue, pour mieux le comprendre et évoluer avec lui, sans vouloir le contraindre ; c'est aussi prétendre profiter de son évolution. C'est apprendre à mieux connaître le système dans lequel nous progressons en ayant recours à une modélisation éclairante, ou encore apprendre à mieux nous connaître en tant que système cognitif intégré dans ce système englobant. En modélisant de la sorte les systèmes qui nous entourent ou qui nous habitent, nos actions deviennent des actes métacognitifs. C'est en « appliquant une méthodologie propre à la démarche systémique que les modèles construits à partir de réalités perçues deviennent la représentation de ce qui est reçu comme et par un système général » [Ibid., p. 37]. De ces considérations, l'objectif clairement poursuivi est formulé comme une invite à modéliser ce qui caractérise un monde réel, propice à l'autoconstruction de la connaissance.

\section{Modélisation d'un ENA perçu comme un système complexe}

Notre article vise principalement à montrer ce que la théorie de la Modélisation des systèmes complexes (Le Moigne, 1999) peut apporter à l'analyse d'un ENA et la manière dont il est possible de la mettre en œuvre concrètement.

Selon la théorie de la modélisation systémique de la complexité (Ibid.), modéliser un système complexe, c'est d'abord modéliser un système d'actions synchroniques ${ }^{11}$ (qui fonctionne), diachronique $^{12}$ (qui se transforme en fonctionnant), téléologiques (qui a une finalité, un but) et récursif (autonomisation) dans un environnement actif. La modélisation systémique passe également par le respect d'une logique conjonctive qui vise à joindre et non disjoindre, d'une part les concepts d'« Environnement Actif» et de «Projet ou de Téléologie», et d'autre part ceux de fonctionnement synchronique «le Faire » et de transformation diachronique «le Devenir ». La procédure cybernétique caractérise la conjonction des deux premiers concepts, la procédure structuraliste la conjonction des deux derniers. Le concept de Système Général (SG) résulte, quant à lui, de la conjonction de ces deux concepts fondateurs à savoir la procédure cybernétique et structuraliste (Fig. 5).

\footnotetext{
${ }^{11}$ Désigne les phénomènes ou processus se produisant en même temps, ne se succédant pas. (source : Le petit lexique des termes de la complexité du « Réseau Intelligence de la complexité » http://www.intelligence-complexite.org/fr/documents/lexique-determes-de-la-complexite.html ${ }^{12}$ Adjectif signifiant ce qui se prolonge, évolue ou se transforme dans le temps (par opposition à synchronique). Même source que
ci-dessus.
} 
I a conjonction systémique conjonction des deux conjonctions

Cybernétique et Structuraliste

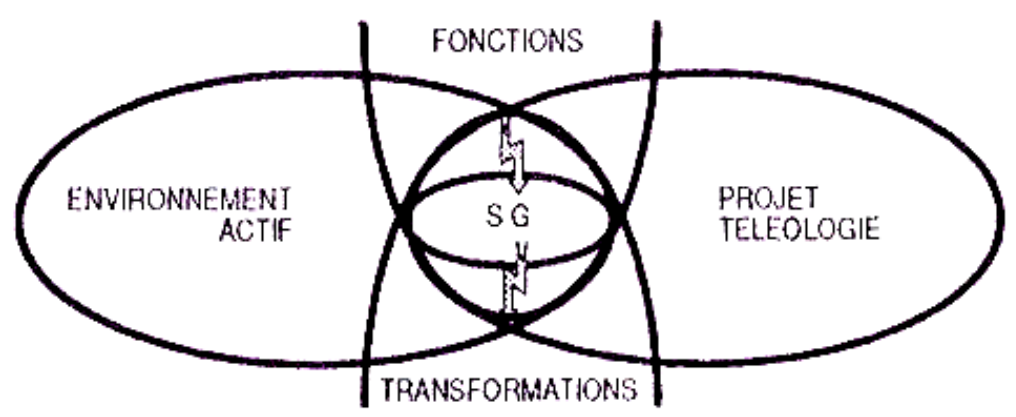

Figure 5. La conjonction systémique [LEM 99, p. 40]

«Tout système complexe peut donc être représenté par un système d'actions multiples, ou par un processus qui peut être un enchevêtrement de processus. Celui-ci peut être représenté par la désignation des fonctions identifiables qu'il exerce ou peut exercer » (Ibid., p. 48).

\subsection{Identification et représentation des processus en jeu dans un ENA}

La première étape de la modélisation systémique que nous proposons correspond au premier niveau $d u$ modèle archétype de l'articulation d'un système complexe à neuf niveaux décrit en détail par Kenneth E. Boulding [BOU 56, 04] ${ }^{13}$ et exploité par la suite par Jean-Louis Le Moigne [LEM 99, p. 58). A cette étape, la perception d'abord syncrétique de l'environnement numérique ou du phénomène à modéliser ne permet pas au modélisateur d'en percevoir les détails. Il fixe alors son contour, ses frontières ${ }^{14}$ avec le monde extérieur et examine sa fonction globale qu'il énoncera par la suite. La finalité du projet est décrite dans sa dimension dynamique.

Découle de cette activité la description des processus imbriqués ou des complexes d'actions lesquelles meubleront progressivement la boîte noire qui représente à la fois le système et son projet global. «Aux familles de projets, on associera des hypothèses de sous-systèmes que l'on cherchera à articuler ... en se référant au projet global du système de modélisation (et non à l'hypothétique nature des choses).

Peraya [PE 03], Meunier et Peraya [MEN 04], et Charlier et al. [CHA 06] se sont intéressés à «l'approche par fonctions constitutives de tout environnement de formation médiatisée » sans pour autant (à notre connaissance) que la théorie de la modélisation des systèmes complexes n'ait été convoquée explicitement. Le cadre d'analyse relatif à ces fonctions constitutives de tout environnement de formation médiatisée constitue aujourd'hui un cadre de référence. Il met en relation huit fonctions retenues à partir des différentes taxonomies que la littérature scientifique propose à ce sujet.

Peraya, Charlier, et Deschryver, [PAE 08, p. 20] citent successivement : «Paquette (1993) ; Collins, Greeno et Resnick (1994); Basque et Doré (1998); de Vries, (2001); Henri et Lundgren-Cayrol (2001); Peraya et Deschryver (2002-2005) ; Gauthier (2004) ». Sur la base de ces citations, ces auteurs proposent de retenir des fonctions «a) d'awareness ou de mise en évidence de « signes de la présence [des acteurs] à distance » (Weissberg, 2003 ; Jacquinot-Delaunay, 2006) ; b) d'interaction sociale qui

\footnotetext{
${ }^{13}$ K.E. Boulding (1967) présente dans Skeleton of Science ( "l'armature de la science ») une analyse en neuf niveaux, que nous développerons et utiliserons par la suite. Elle a très largement contribué à éclairer les conditions d'utilisation de l'approche ou de la méthodologie systémique.
}

${ }^{14}$ Ce pourrait aussi être les limites « ce monde » pour les MOOC, par exemple. 
regroupe ce qui est classiquement désigné comme collaboration, communication et échanges ; c) d'information définie au sens de mise à disposition de ressources ou d'objets ; d) de production (individuelle ou collective) ; e) de gestion et de planification (des activités et des acteurs) ; f) de soutien et d'accompagnement; g) d'émergence et de systématisation de l'activité métaréflexive ; h) d'auto et d'hétéro-évaluation » (Peraya, Charlier, et Deschryver, 2008 , p. 20).

Les huit fonctions ainsi formalisées peuvent être particulièrement utiles au cours de cette première étape de modélisation systémique de la complexité ; elles ont d'ailleurs été en partie utilisées par Peraya et Bonfils [PER 14, p. 13] lors d'une étude conduite sur les EPA (Environnement Personnel d'Apprentissage).

Les termes et légendes peuplant la carte conceptuelle du système ainsi construite et servant à nommer chacun des traits perçus du phénomène à modéliser sont déterminés par itérations successives. Le modélisateur complète cette carte en franchissant un à un les neuf niveaux fonctionnels du modèle archétype. "L'argument consiste à considérer une sorte de complexification progressive de la modélisation systémique » [Ibid., p.58]. Les ajustements se font par itérations successives pour réduire le plus possible la distance entre une représentation construite par le modélisateur et la représentation des utilisateurs [LEM 08, p. 105].

\subsection{Des processus finalisés dans un environnement actif (le système fait)}

Les processeurs inscrits au cœur de la carte conceptuelle sont ensuite exploités tour à tour en tenant compte des actions qu'ils produisent, c'est-à-dire en explicitant comment les valeurs d'entrée (les intrants) de chaque processeur sont transformées pour devenir des valeurs de sortie (les extrants) ${ }^{15}$. Modéliser un environnement actif, c'est modéliser l'activité qui s'y exerce par l'accomplissement d'actions, de transactions et d'interactions. Cette étape correspond au deuxième niveau de complexification du modèle archétype.

Pour rendre compte simultanément de ces actions et contre-réactions, autrement dit de ces interrelations d'actions (réseau et feed-back), nous suggérons, d'une part, (i) de signifier leur présence active en consignant dans une matrice structurelle leur origine et leur destination et, d'autre part, (ii) de dresser pour chacune d'elles l'inventaire des valeurs d'entrée et de sortie. Et pour traduire l'action et la régulation du système à un instant donné (ce que le système fait) qui correspond au troisième niveau de complexification du modèle archétype, nous proposons (iii) de construire un diagramme à flots de données qui nous parait être un bon moyen de montrer les dépendances fonctionnelles

\subsection{Perception dynamique des processus (le système évolue).}

Nous abordons ensuite le niveau 4 de complexification du modèle archétype, non plus de manière statique (le système «fait») mais selon sa composante dynamique (le système «devient »). Nous décrirons alors les séquences typiques des processus (scénarii) en mettant en évidence les évènements qui activent les actions. Les traces laissées par ces événements nous sont utiles pour élaborer les diagrammes d'états des processus.

\subsection{Un système capable de décider, de traiter et mémoriser l'information et de se coordonner}

Un cinquième niveau vient s'ajouter aux étapes de complexification de la modélisation systémique. Le système devient capable de décider son activité, traiter l'information qu'il produit et prendre des décisions sur son propre comportement.

Ce cinquième niveau marque une étape importante dans la progressivité du processus de complexification à neuf niveaux. Les quatre premiers niveaux caractérisent la conjonction des

\footnotetext{
${ }^{15}$ Remarquons dès à présent qu'un diagramme à flux de données du modèle fonctionnel de la modélisation orientée objet que nous allons utiliser pour la circonstance « montre comment les valeurs sortantes sont dérivées à partir de valeurs entrantes » (Rumbaugh, 1995, p.124). 
procédures cybernétique et structuraliste : le système a un projet dans un environnement actif, il existe, fait, et se transforme. Les niveaux suivants révèlent d'abord son aptitude à générer, traiter et mémoriser l'information (niveau 5 et 6). Puis ils le reconnaissent capable de se coordonner (niveau 7) et d'élaborer de nouveaux projets, de nouvelles formes d'actions, de faire preuve d'imagination (niveau 8). Enfin le processus actif développe une capacité d'autofinalisation qui lui permet de décider de son devenir, de faire des choix sur ses propres orientations (niveau 9).

\section{Les méthodes et langages de modélisation}

Pour faciliter d'un point de vue méthodologique la construction du modèle, nous suggérons au modélisateur d'adopter, à différentes étapes de son élaboration (modèle fonctionnel, dynamique, ...), une méthode qui s'inspire de la modélisation « orientée objet ${ }^{16}$ (OMT) élaborée par Rumbaugh ${ }^{17}$ et al. [RUM 95]. Dans cette approche, un «objet » est conceptuellement très différent d'un simple constituant du système. Il représente un concept, une idée ou toute entité du monde physique et non pas seulement un constituant statique ou un organe du système. Et si l'objet peut être un concept, alors un processus peut être un objet selon cette acception ${ }^{18}$.

Dans cette perspective, nous observons aussi qu'il existe des langages de modélisation en lien avec la méthode OMT qui proposent toute une série de représentations symboliques qui permettent de représenter graphiquement les processus en jeu dans un système complexe. Elles sont généralement accessibles à partir d'une bibliothèque propre au logiciel utilisé. Nous préconisons en outre d'utiliser le langage UML (Unified Modeling Language) pour modéliser ces processus. De nombreuses représentations sont proposées dans les ouvrages de Trestini [TRE 18a; TRE 18b] afin d'illustrer concrètement cette démarche. Nous proposons néanmoins en figure 6 une représentation de la fonction «Interractions sociales » de niveau 2 dans le cas de la modélisation d'un MOOC $^{19}$ pris en exemple à des fins illustratives.

\footnotetext{
${ }^{16}$ Objet Modeling Technique.

17 James Rumbaugh est le créateur du langage de " modélisation orienté objet ». Il est également l'un des trois pères du langage UML, avec Grady Booch et Ivar Jacobson. (Source : I'encyclopédie en ligne Wikipedia).

${ }^{18}$ Sfard (1992) nomme " réification des processus » la transition de la conception « processus » à la conception « objet ». L'au teur a également souligné que les termes processus et objet devraient être compris comme différentes facettes de la même notion plutôt que comme composants séparés. Autrement dit, les deux aspects opérationnel et structurel sont complémentaires (tiré de http://didel.script.univ-parisdiderot.fr/claroline/backends/download.php?url=LORvY3NfZGI2ZXJzL01pbmhfQ0pTTVRFX1ByZXByaW50LnBkZg\%3D\%3D\&cidReset= true\&cidReq=TICE LDAR)

${ }^{19}$ Il s'agit de la modélisation du MOOC « Gestion de Projet » de Rémi Bachelet.
} 


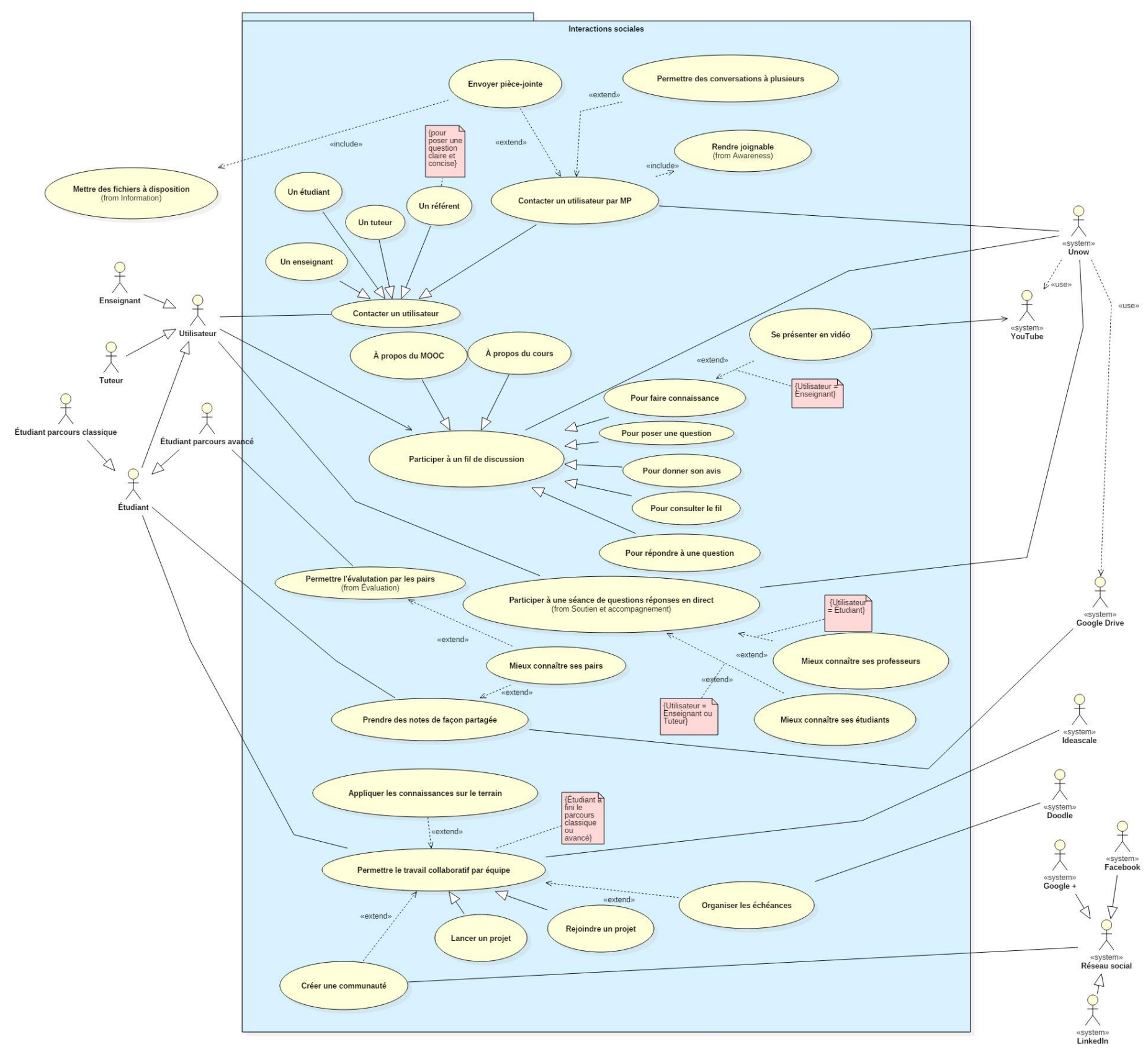

Figure 6. Cas d'utilisation : Intéraction sociale

\section{Conclusion}

Ces quelques exemples nous ont permis d'illustrer la manière dont se modifient les modèles pour répondre à un changement de contexte sociotechnique et en particulier lorsque s'y introduisent des innovations techno-pédagogiques. Les différentes adaptations apportées aux modèles existants sont nées de différents besoins. Il a ainsi pu arriver qu'un concept nécessaire à l'étude (conflit instrumental par exemple) n'ait pu être étudié du fait de son accessibilité difficile (ou de sa mauvaise lisibilité) à travers le modèle utilisé. A d'autres occasions, il nous a été difficile de représenter des communautés d'acteurs très différentes les unes des autres (dans un ENT scolaire par exemple : enfant, parents, enseignants) agissant ensemble au cœur d'un ENA. Par ailleurs, le modèle sur lequel nous nous basions devait être compatible avec différents points de vue issus de différentes théories développées autour de l'activité instrumentée (genèse instrumentale, socioconstructivisme, etc.) : cette contrainte est une autre source d'évolution des modèles.

Avec notamment l'arrivée des pédagogies actives dans le e-learning, nous sommes passés d'une logique de cours enseigné, centré sur les contenus et les apprentissages prescrits, à une logique centrée sur l'apprentissage autonome. Les modèles, d'abord fondés sur la mise à disposition de ressources, ont 
donc été progressivement remplacés par des modèles d'activités pédagogiques pour ensuite englober l'ensemble des activités comme celles de gestion par exemple. Ainsi, nous sommes assez rapidement passés d'un modèle de scénarisation des apprentissages à un modèle de dispositif de formation intégrant toutes les activités qui y règnent.

Nous avons voulu montrer dans cette communication le cheminement réflexif qui nous a conduit à mener une recherche sur les ENA de dernière génération $(\mathrm{OCW}, \mathrm{MOOC}, \mathrm{RSN}, \ldots)$. Les techniques de modélisation systémique de la complexité y ont été à l'évidence privilégiées. Rappelons que «la systémique se définit comme la science qui se donne pour objet la conception et l'analyse de modèles de phénomènes complexes» [LEC 06, 39 de paragraphe]. La modélisation s'appuie sur une représentation qui permet une compréhension à la fois individuelle et commune de l'objet étudié ; nous avons bien conscience que la connaissance de cet objet ne pourra jamais décrire totalement une quelconque réalité. Nous avons cependant reconnu dans la modélisation systémique de la complexité un moyen efficace d'appréhender la complexité des ENA mais également un moyen privilégié de découvrir certains phénomènes émergents dont l'apprentissage fait partie. Nous espérons pouvoir tirer de ces techniques quelques éléments de prospective très attendus de nos politiques publiques ${ }^{20}$ en quête d'indicateurs sur l'évolution de ces dispositifs innovants. Mais ne nous y trompons pas ; il ne s'agit pas de vouloir réduire l'incertitude pour maîtriser la complexité. Il s'agit plutôt de chercher à la gérer, à en découvrir ses effets et apprendre à les anticiper. «Traiter de situations complexes relève du partage de connaissances, de processus d'apprentissage et d'explorations qui ouvrent de nouveaux espaces à l'action, en modifiant à la fois la dimension cognitive (la compréhension de la situation) et la dimension pratique (les actions menées) : la situation change parce que nous changeons avec elle $^{21}$ » [HUB 15, p. 96].

Dès lors qu'il est perçu comme un phénomène complexe, le parti pris de cette communication est donc de considéré qu'un ENA de dernière génération peut être parfaitement représenté et analysé dans le paradigme de la Modélisation systémique de la complexité. La célèbre formule « le tout est plus que la somme des parties » de Von Ehrenfels ${ }^{22}$ montre que peuvent émerger de l'activité de modélisation des propriétés nouvelles caractérisant le système et résultant de l'agrégation et de la rétroaction de ses éléments constitutifs. De là vient d'ailleurs le concept d'émergence qui « rejette ainsi la possibilité que la connaissance globale d'un phénomène puisse uniquement résulter de la simple connaissance de ses composants fondamentaux $»^{23}$.

\section{Bibliographie}

[ANC 92] ANCORI B., « Mémoire et apprentissage : de la neurobiologie à l'auto-organisation », dans B. ANCORI (DIR.), Apprendre, se souvenir, décider. Une nouvelle rationalité de l'organisation, p. 51-104, CNRS Editions, Paris, 1992.

[ANI 06] ANIORTE P., CARIOU E., GouARderes E., «Modélisation de systèmes complexes distribués : l'ingénierie des modèles pour l'intégration des paradigmes "agent" et "composant" », Journée Multi-Agent et Composant (JMAC 2006), Nîmes, France, mars 2006, disponible à l'adresse : http://csl.ensmdouai.fr/MAAC/uploads/anioriteJMAC2006. pdf

[ATL 79] ATLAN H., Entre le cristal et la fumée. Essai sur l'organisation du vivant, Le Seuil, Paris, 1979.

[ATL 98] ATLAN H., «La fin du "tout génétique" ? Vers de nouveaux paradigmes en biologie », Conférence-débat organisée par le groupe Sciences en questions, INRA, Paris, France, mai 1998.

[BAT 77] BATESON G., Vers une écologie de l'esprit, Le Seuil/Essais/Points, 1977.

\footnotetext{
${ }^{20}$ Parmi les nombreuses définitions de politique publique nous retiendrons celle-ci : « Une politique publique est ce que les gouvernements choisissent de faire ou de ne pas faire » (Source : https://fr.scribd.com/doc/46489659/Politiques-publiquesDefinition-et-objet)

${ }^{21}$ Hatchuel, A., Weil, B., 2009. C-K design theory: an advanced formulation, Research in Engineering Design, $19(4), 181-192$.

${ }^{22}$ Christian von Ehrenfels (1859-1932) serait en effet l'auteur de cette formule célèbre selon Louis-José Lestocart : http://www.tribunes.com/tribune/alliage/59/page8/page8.htm/
}

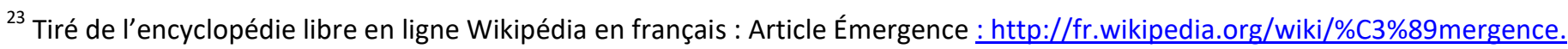


[BEG 13] BEGUIN S., Quelle différence entre complexe et compliqué ?, blogue, 10 janvier 2013, disponible à l'adresse: http://www.simplixi.fr/difference-complexe-et-complique/.

[BLA 95] BLACKLER, F., " Knowledge, knowledge work and organizations: An overview and interpretation », Organization Studies, vol. 16, n 6, pp. 1021-1046

[BOU 56, 04] BoulDING K.E., "General systems theory, the skeleton of science », dans E:CO Special Double Issue, vol. 6, n 1-2, p. 127-139, 2004, disponible à l'adresse : http://pespmc1.vub.ac.be/books/Boulding.pdf.

[CHA 06] CHARLIER B., DESChryver N., et al., «Apprendre en présence et à distance : une définition des dispositifs hybrides », Distances et savoirs, vol. 4, n 4, p. 469-496, 2006, disponible à l'adresse : http://archiveouverte.unige.ch/unige:17649 (consulté le 26 décembre 2015).

[DAN 05] Daniellou, F. ET RABARDEl, P., "Activity-oriented approaches to ergonomics : Some traditions and communities". Theoretical Issues in Ergonomics Science, 6(5) : 353-357, 2005.

[DUC 05] DUCROCQ-HENRY, S., « Le Lan Party, Aspects de socialisation, d'identité et d'apprentissage reliés au jeu vidéo en réseau », Conférence du Professeur au département d'éducation de l'UQAT, Canada, juin 2005, disponible à l'adresse : http://www.ludisme.com/media/Conference_SDH_LAN.pdf.

[ENG 87] Engeström Y., Learning by Expanding: An Activity-theoretical Approach to Developmental Research, OrientaKonsultit Oy, Helsinki, 1987.

[FEL 17] FELDER, J., "Comprendre les processus de construction et de régulation des EPA par des étudiants universitaires », Sticef - Vol. 24, n³, 2017 (paragraphe 5.1.3)

[FOR 05] FORTIN R., Comprendre la complexité. Introduction à la Méthode d'Edgar Morin, Les presses de l'université Laval, Québec, 2005.

[HUB 15] Hubert, B., Aubertin, C., et Billaud, J.-P., Connaître ? Agir ? Interagir ? Natures Science Sociétés, 23(2), 9596, 2015. Récupéré de http://www.nss-journal.org/articles/nss/pdf/2015/03/nss150032.pdf

[JON 91] JONASSEN D.H., Handbook of research for educational communications and the technology, Simon \& Schuster Macmillan, New York, 2009.

[KUU 96] KUUTTI K., « Activity Theory as a Potential Framework for Human-Computer Interaction Research », dans B. NARDI (DIR.), Context and consciousness: Activity theory and human computer interaction, p. 17-44, MIT Press, Cambridge, MA, 1996.

[LEC 06] LECAS G., «La modélisation systémique : outils méthodologiques pour économistes », Innovations, vol. 2, $\mathrm{n}^{\circ}$ 24, p. 199-230, 2006, Disponible à l'adresse : https://www.cairn.info/revue-innovations-2006-2-page-199.htm.

[LEG 04] LEGENDRE M.-F., « Approches constructivistes et nouvelles orientations curriculaires : d'un curriculum fondé sur l'approche par objectifs à un curriculum axé sur le développement de compétences », dans p. JONNAERTP et D. MASCIOTRA (DIR.), Constructivisme, choix contemporains : hommage à Ernst Von Glasersfeld, p. 51-87, Presses de l'université du Québec, Sainte-Foy, Québec, 2004.

[LEM 08] LEMIRE G., Modélisation et construction des mondes de connaissances: Aspects constructiviste, socioconstructiviste, cognitiviste et systémique, Les Presses de l'université Laval, Québec, 2008.

[LEM 99] LE MOIGNE J.-L., La modélisation des systèmes complexes, (original 1990), Dunod, Paris, 1999.

[LEO 81] LÉONTIEV A.N., « The problem of activity in psychology », dans J.V. WERTSCH (DIR.), The concept of activity in soviet psychology, M.E. Sharpe Inc., Armonk, NY, 1981.

[LEW 98] LEWIS R., « Learning together: a rationale, some experiences and a framework », dans J.-F. ROUET et B. DE LA PASSARDIERE, Actes du 4ème colloque Hypermédias et Apprentissages, (trad par J.-F. ROUET), Poitiers, France, 15, 16 et 17 octobre 1998 .

[MAR 11] MARQUET M., «eLearning et conflit instrumental : entre didactique, pédagogie et technique », Recherche et Formation, n $^{\circ} 68$, p. 31-46, 2011, disponible à l'adresse : http://rechercheformation.revues.org/1499 (consulté le 6 décembre 2015).

[MIT 09] Mitchell M., Complexity, guided tour (349), Oxford University Press, Oxford, 2009.

[MEU 04] MEUNIER J.P., PERAYA D., Introduction aux théories de la communication. Analyse sémio-pragmatique de la communication médiatique, De Boeck, Bruxelles, 2004

[NAR 96] NARDI, B. A., Context and Consciousness : Activity theory and human compter interaction. Cambridge, MA : MIT Press, 1996.

[PER 03] PERAYA D., « De la correspondance au campus virtuel : formation à distance et dispositifs médiatiques », dans B. Charlier et D. Peraya, (dir.), Technologie et innovation en pédagogie. Dispositifs innovants de formation pour l'enseignement supérieur, p. 79-92, De Boeck, 2003, disponible à l'adresse: http://archiveouverte.unige.ch/unige:29016. 
[PER 05] PERAYA D., «La formation à distance : un dispositif de formation et de communication médiatisées. Une approche des processus de médiatisation et de médiation», TDR, $\mathrm{n}^{\circ} 0 \mathrm{a}, \mathrm{p} .12,2005$, disponible à l'adresse : http://www.revue-tice.info/document.php?id=520.

[PER 08] Peraya D., Charlier B., Deschryver N., « Dispositifs hybrides », dans J.-P Pernin, et H. Godinet, (dir), ACI «Terrains, Techniques, Théories», Projet ACTEURS (Activités Collectives et Tutorat dans l'Enseignement Universitaire: Réalités, Scénarios et usages des TICE), INRP, Lyon, p. 14-24, 2008, disponible à l'adresse : http://eductice.ens-lyon.fr/EducTice/recherche/archives/acteurs/RapportProjetACTEURS. pdf.

[PER 14] PERAYA D., BonfILS P., «Détournements d'usages et nouvelles pratiques numériques : l'expérience des étudiants d'Ingémédia à l'université de Toulon », Sticef, $\mathrm{n}^{\circ} 21,2014$, disponible à l'adresse : http://sticef.univlemans.fr/num/vol2014/19-peraya-epa/sticef_2014_NS_peraya_19.htm\#fn4.

[RAB 95] RABARDEL P., Les hommes et les technologies. Approches cognitives des instruments contemporains, A. Colin, Paris, 1995.

[RUM 95] Rumbaugh J., Blaha M., Premerlani W., EdDy F. ET LoREnSen,W., Modélisation et conception orientées objet, Masson, Paris, 1995.

[SIE 05] SIEMENS G., «Connectivism: A learning theory for the digital age », International Journal of Instructional Technology and Distance Learning, vol. 2, $\mathrm{n}^{\circ} 1, \quad 2005$, disponible à l'adresse : http://www.itdl.org/Journal/Jan_05/article01.htm.

[SNO 02] SNOWDEN D., «Complex acts of knowing: paradox and descriptive self-awareness », Journal of Knowledge Management, vol. 6, n 2, p. 100-111, 2002.

[TAR 86] Tardieu H., Rochfeld A., Colletti R., La Méthode Merise, Principes et outils, Les éditions d'Organisation, Paris, 1986.

[TRE 08] TRESTINI M., «Préface », dans G. Lemire, (dir.), Modélisation et construction des mondes de connaissances : Aspects constructiviste, socioconstructiviste, cognitiviste et systémique, Les Presses de l'université Laval, Québec, p. 1-8, 2008, disponible à l'adresse : http://www.pulaval.com/catalogue/modelisation-construction-des-mondesconnaissances-aspects-9187.html.

[TRE 09b] TRESTINI M., « Préface », dans G. Lemire (dir.), Modélisation et construction des mondes de connaissances : Aspects constructiviste, socioconstructiviste, cognitiviste et systémique, Cahier des Lectures MCX (Modélisation de la Complexité) [en ligne sur proposition de Jean-Louis Le Moigne], janvier 2009, disponible à l'adresse: http://www.intelligence-complexite.org/fr/cahier-des-lectures/recherche-dune-note-de-

lecture.html?tx_mcxapc_pi1\%5Baction\%5D=noteDetail\&tx_mcxapc_pi1\%5BidNote\%5D=711\&cHash=5562b2fabb6 $62770 \mathrm{~b} 1 \mathrm{ff} 919 \mathrm{c} 52 \mathrm{~b} 86326$.

[TRE 10a] TRESTINI M., LEMIRE G., «Environnements Constructivistes d'Apprentissage : quand les activités collectives et individuelles permettent aux apprenants de construire leurs connaissances », Education Canada, vol. 50, n ${ }^{\circ}$ 1, p. 5660, 2010, disponible à l'adresse : https://www.edcan.ca/wp-content/uploads/EdCan-2010-v50-n1-Trestini.pdf.

[VAL 75] VAlery P., Cahiers, Encyclopédie de la Pléiade, Gallimard, Paris, 1975.

[VON 56] Von Bertalanffy L., General System Theory, General Systems Yearbook 1, 1:10, Brazziler, New York, 1956.

[VYG 85] VYGOTSKI L., «La méthode instrumentale en psychologie », dans J.-P. Bronckart et B. Schneuwly, Vygotski aujourd'hui, Delachaux \& Niestlé, Paris, 1985. 